\title{
Decision Procedure for a Fragment of Mutual Belief Logic with Quantified Agent Variables
}

\author{
Regimantas Pliuškevičius and Aida Pliuškevičienė \\ Institute of Mathematics and Informatics \\ Akademijos 4, Vilnius 08663, LITHUANIA \\ \{regis, aida\}@ktl.mii.lt
}

\begin{abstract}
A deduction-based decision procedure for a fragment of mutual belief logic with quantified agent variables $(M B Q L)$ is presented. A language of $M B Q L$ contains variables and constants for agents. The language of $M B Q L$ is convenient to describe properties of rational agents when the number of agents is not known in advance. The multi-modal logic $K D 45_{n}$ extended with restricted occurrences of quantifiers for agent variables is a component of $M B Q L$. For this logic loop-check-free sequent calculus is proposed. This calculus corresponds to contraction-free calculus and does not require to translate sequents in a certain normal form. Another new point of presented decision procedure is existentially invertible separation rules. For a sequent containing occurrences of mutual belief modality two type of loop-check can be used: for positive occurrences of mutual belief modality loop-check can be used to find non-logical (loop-type) axioms, and for negative ones - to establish a non-derivability criterion.
\end{abstract}

\section{Introduction}

Mutual belief (common knowledge) logics are multi-modal logics extended with mutual belief (common knowledge) and everybody believes (everybody knows) modalities. Sequent-like calculi (with analytic cut rule instead of looptype axioms) and Hilbert-style calculi for propositional common knowledge logics (based on finite set of agents) are constructed in several works (see, e.g., [1], [4], [11]). In [6] Hilbert-style calculus for common knowledge logic with infinite set of agents is presented. This calculus involves some restrictions on cardinality of set of agents and contains rather complex axiom for everybody knows operator. Propositional Hilbert-type calculus for mutual belief logic (based on finite set of agents) is constructed in several works (see, e.g., [2]).

Propositional agent-based logics are often insufficient for more complex real world situations. First-order extensions of these logics are necessary whenever a cardinality of an application domain and/or the number of agents are not known in advance. In [14] it is described a rich logic LORA (Logic of Rational Agents), based on a three-sorted first-order logic (containing variables for agents, actions and other individuals), multi-agent $B D I$ logic, and a dynamic 
logic. In [10] a logic $Q L B$ (quantified logic for belief) with Barcan axiom containing variables for agents and other individuals is presented. The same idea as in [10] and [14], namely, use of term as an agent, is utilized in term-modal logics [5]. In [13] a decision procedure for a fragment of temporal logic of belief and actions with restricted occurrences of quantified agent and action variables is presented.

In this paper, a fragment of mutual belief logic with quantified agent variables $(M B Q L)$ is considered. Different from [5], [10] and [14], the language of $M B Q L$ does not contain function symbols. The aim of this paper is to present a deduction-based decision procedure for $M B Q L$. The presented decision procedure is based on sequent-like calculus $M B Q$ with invertible rules (in some sense). Separation rules is an important point of presented decision procedure. These existentially invertible rules incorporate "bad" quantifier rules for agent variables, the rules for everybody believes modality, and rules for belief modalities. Some deduction tools similar to separation rules are used informally in [12] for propositional (single agent) $B D I$ logic. A decision procedure for logic $K D 45_{n}$ extended with restricted occurrences of quantifiers for agent variables is another important point. For this logic loop-check-free sequent calculus is proposed. This calculus corresponds to contraction-free sequent calculus. However, loop-check-free type sequent calculus differs from contraction-free sequent calculus. In contraction-free sequent calculus (see [3], [7]) duplication of the main formula in the premise of a rule is eliminated at all. In loop-check-free sequent calculus duplication of the main formula in the premise of a rule is not eliminated but applications of rules containing such duplications are restricted. It allows to eliminate loop-check and does not require to translate sequents in a certain normal form as in [7]. For a sequent containing occurrences of mutual belief modality two type of loop-check can be used: for positive occurrences of mutual belief modality loop-check can be used to find non-logical (loop-type) axioms, and for negative ones - to establish a non-derivability criterion.

Here a procedural approach of decidable logical calculi is used and we assume that the notions of a decidable calculus and a deduction-based decision procedure are identical.

The paper is organized as follows. In Section 2, the language and the semantics of the $M B Q L$ are presented. In Section 3, auxiliary tools for the presented decision procedure are described. In Section 4, a decision algorithm is presented relying on the sequent calculus $M B Q$ and some examples demonstrating the presented algorithm are given. In Section 5, a foundation of the decision algorithm is given. 


\section{Language and semantics of $M B Q L$}

The $M B Q L$ consists of the multi-modal logic $K D 45_{n}$ (doxastic logic or weak$S 5_{n}$ ) extended with restricted occurrences of quantifiers for agent variables and logic containing mutual belief and everybody believes modalities [2].

The language of $M B Q L$ contains: (1) a set of propositional symbols $P$, $P_{1}, \ldots, Q, Q_{1}, \ldots ;(2)$ a set of agent constants $i, i_{1}, \ldots, a_{1}, \ldots, b_{1}, \ldots,\left(i, i_{l}\right.$, $\left.a_{j}, b_{j} \in\{1, \ldots\}\right)$; (3) a set of agent variables $x, x_{1}, \ldots, y, y_{1}, \ldots$; (4) a set of belief modality of the shape $\mathbf{B}(t)$, where $t$ is an agent term, i.e., an agent constant or an agent variable; everybody believes modality EB; mutual belief modality MB; (5) logical operators: $\supset, \wedge, \vee, \neg, \forall, \exists$.

Formula of $M B Q L$ is defined inductively as follows: every propositional symbol is formula; if $A, B$ are formulas, then $A \supset B, A \wedge B, A \vee B, \neg(A)$ are formulas; if $i$ is an agent, $A$ is a formula, then $\mathbf{B}(i) A$ is a formula; if $x$ is an agent variable, $A$ is a formula, $Q \in\{\forall, \exists\}$, then $Q x \mathbf{B}(x) A$ is a formula; if $A$ is a formula, then $\operatorname{EB}(A)$ and $\operatorname{MB}(A)$ are formulas. The formula $A$ is a logical one if $A$ contains only logical operators and propositional symbols.

As it follows from definition of formula, we do not consider, for example, expressions of the shape $\forall x \exists y \mathbf{B}(x) \mathbf{B}(y) A$, but expressions of the shape $\forall x \mathbf{B}(x) \exists y \mathbf{B}(y) A$ are considered.

When the formula under consideration contains occurrences of operators $\mathbf{E B}$ and/or $\mathbf{M B}$ it is assumed that the number of agents is finite. In this case the formula $\forall x \mathbf{B}(x) A$ means informally the same as the formula $\bigwedge_{i=1}^{n} \mathbf{B}(i) A$ and the formula $\exists x \mathbf{B}(x) A$ - as the formula $\bigvee_{i=1}^{n} \mathbf{B}(i) A$. Since the exact number of agents is not known in advance, in general, we use formulas with quantified agent variables.

The formula $\mathbf{B}(i) A$ means "agent $i$ believes $A$ ". Formal semantics of the formula $\mathbf{B}(i) A$ satisfies the semantics of the logic $K D 45_{n}$. The formula $\mathbf{E B}(A)$ means "every agent believes $A$ ", i.e. $\mathbf{E B}(A) \equiv \bigwedge_{i=1}^{n} \mathbf{B}(i) A$. The formula $\operatorname{MB}(A)$ means: " $A$ is mutual belief of all agents". Therefore we use only socalled public mutual belief modality and assume that there is perfect communication between agents. The formula $\mathbf{M B}(A)$ has the same meaning as the infinite formula $\bigwedge_{k \geq 1} \mathbf{E B}^{k}(A)$, where $\mathbf{E B}^{1}(A)=\mathbf{E B}(A)$, and $\mathbf{E B}^{k}(A)=$ $\mathbf{E B}^{k-1}(\mathbf{E B}(A))$, if $k>1$. Infinitary nature of the modality $\mathbf{M B}$ is explained in [14]. The modalities $\mathbf{M B}$ and $\mathbf{E B}$ behave as modality of logic KD4. In addition, these modalities satisfy an induction-like property:

$$
\mathbf{E B}(A) \wedge \mathbf{M B}(A \supset \mathbf{E B}(A)) \supset \mathbf{M B}(A) .
$$

All belief modalities can be nested. For example, formula $\mathbf{B}\left(i_{1}\right) \mathbf{B}\left(i_{2}\right) P$, where $P$ is a proposition "John is a good programmer", means "agent $i_{1}$ believes that agent $i_{2}$ believes that John is a good programmer". The formula 
$\exists x \mathbf{B}(x) \forall y \mathbf{B}(y) P$, where $P$ means the same as above, means "some agent believes that each agent believes that John is a good programmer".

To define the formal semantics of the formula $Q x \mathbf{B}(x) A(Q \in\{\forall, \exists\})$ we must present an interpretation of agent variables. Such interpretation is received by means of an assignment: $V \rightarrow D$ (agent assignment), where $V$ is a set of agent variables, $D$ is a domain of agent constants. A model $M$ is a pair $<\mathcal{I}, \mathbf{a}\rangle$, where $\mathbf{a}$ is an agent assignment, $\mathcal{I}$ is a tuple $\langle D, S t, \pi, \mathbf{R}\rangle$, where $D$ is a domain of agent constants; $S t$ is a set of states; $\pi$ is an interpretation function of the propositional variables; $\mathbf{R}$ is the accessibility relations. All these relations satisfy transitive, serial, and Euclidean properties.

The concept "formula $A$ is valid in $M=<\mathcal{I}$, a $>$ at the state $s \in S t$ " (in symbols $M, s \models A$ ) is defined by induction on the structure of the formula of $M B Q L$. Let us define only the cases when $A$ is $Q x \mathbf{B}(x) N$, where $Q \in\{\forall, \exists\}$ (other cases are defined analogously as in [2], [4], [11], [14]).

$M, s=\forall x \mathbf{B}(x) N$ if and only if for every agent assignment $\mathbf{a}^{\prime}$ which differs from a at most with respect to an agent constant $i,\left\langle\mathcal{I}, \mathbf{a}^{\prime}>\models \mathbf{B}(i) N\right.$;

$M, s \models \exists x \mathbf{B}(x) N$ if and only if for some agent assignment $\mathbf{a}^{\prime}$ which differs from a at most with respect to an agent constant $i,\left\langle\mathcal{I}, \mathbf{a}^{\prime}>\models \mathbf{B}(i) N\right.$;

Along with formulas we consider sequents, i.e., formal expressions $A_{1}, \ldots$, $A_{k} \rightarrow B_{1}, \ldots, B_{m}$ where $A_{1}, \ldots, A_{k}\left(B_{1}, \ldots, B_{m}\right)$ is a multiset of formulas. The sequent is interpreted as the formula $\bigwedge_{i=1}^{k} A_{i} \supset \bigvee_{j=1}^{m} B_{j}$. A sequent $S$ is a logical one if $S$ contains only logical formulas.

Let us recall the notions of positive and negative occurrences.

A formula (or some symbol) occurs positively in some formula $B$ if it appears within the scope of no negation sign or in the scope of an even number of the negation sign, once all the occurrences of $A \supset C$ have been replaced by $\neg A \vee C$; in the opposite case, the formula (symbol) occurs negatively in $B$. For a sequent $S=A_{1}, \ldots, A_{k} \rightarrow B_{1}, \ldots, B_{m}$ positive and negative occurrences are determined just like for the formula $\bigwedge_{i=1}^{k} A_{i} \supset \bigvee_{j=1}^{m} B_{j}$.

\section{Some Auxiliary Tools of the Decision Algorithm}

A presented decision procedure is based on a sequent calculus with invertible rules. All derivations are constructed as a backward derivations. In this section, we present the main auxiliary tools of the decision algorithm: logical calculus, reduction and separation rules, and contraction rules.

Let $(j)$ be any rule of a sequent calculus. Rule $(j)$ is applied to get the conclusion of $(j)$ from the premises of $(j)$. If rule $(j)$ is backward applied, i.e., to get premises of $(j)$ from the conclusion of $(j)$ we have a "bottom-up application of $(j)$ " instead of "application of $(j)$ ". The rule $(j)$ is called invertible in a 
sequent calculus $I$, if the derivability in $I$ of the conclusion of $(j)$ implies the derivability in $I$ of each premise of $(j)$. If the rule $(j)$ is invertible, the bottomup application of $(j)$ preserves the derivability.

A decidable calculus $\log$ is defined by the axiom: $\Gamma, A \rightarrow \Delta, A$ (where $A$ is the main formula of the axiom) and traditional invertible rules for logical operators $\supset, \vee, \wedge, \neg$.

A derivation in the calculus $\log$ is constructed as a tree using the bottomup applications of the rules. A derivation $D$ is successful if each leaf of $D$ is an axiom and unsuccessful if there exists a leaf which is not an axiom.

Let us define reduction rules by means of which a sequent is reduced to a set of sequents in some canonical forms (see below).

Reduction rules consist of the following rules:

- Logical rules: all the rules of the calculus $\log$ and the following rules:

$$
\frac{\Gamma \rightarrow \Delta, A[b / x]}{\Gamma \rightarrow \Delta, \forall x A}(\rightarrow \forall) \quad \frac{A[b / x], \Gamma \rightarrow \Delta}{\exists x A, \Gamma \rightarrow \Delta}(\exists \rightarrow),
$$

where the variable $x$ is agent variable and agent constant $b$ (called an eigenconstant) does not enter the conclusion of the rules.

- Rules for mutual belief:

$$
\begin{gathered}
\frac{\mathbf{E B}(A), \mathbf{E B}(\mathbf{M B}(A)), \Gamma \rightarrow \Delta}{\operatorname{MB}(A), \Gamma \rightarrow \Delta}(\mathbf{M B} \rightarrow) \\
\frac{\Gamma \rightarrow \Delta, \mathbf{E B}(A) ; \Gamma \rightarrow \Delta, \mathbf{E B}(\mathbf{M B}(A))}{\Gamma \rightarrow \Delta, \mathbf{M B}(A)}(\rightarrow \mathbf{M B}) .
\end{gathered}
$$

- Rule for everybody believes:

$$
\frac{\Gamma \rightarrow \Delta, \bigwedge_{i=1}^{n} \mathbf{B}(i) A}{\Gamma \rightarrow \Delta, \mathbf{E B}(A)}(\rightarrow \mathbf{E B}), \text { where } n \text { is a number of agents. }
$$

Remark 1 We do not introduce reduction rule for everybody believes operator (corresponding to implication $\mathbf{E B}(A) \supset \bigwedge_{i=1}^{n} \mathbf{B}(i) A$, where $n$ is a number of agents) because it is included in separation rules (see below).

To define the separation rules some canonical forms of sequents are introduced. A sequent $S$ is a primary sequent, if $S$ is of the following shape:

$\Sigma_{1}, \forall \mathcal{B} \Gamma, \mathbf{E B} \Pi_{1}, \mathbf{M B} \Delta_{1} \rightarrow \Sigma_{2}, \exists \mathcal{B} \Delta, \mathbf{E B} \Pi_{2}, \mathbf{M B} \Delta_{2}$, where

- for every $i(i \in\{1,2\}) \Sigma_{i}$ is empty or consists of logical formulas;

- $\forall \mathcal{B} \Gamma$ denotes a list $\forall x \mathbf{B}(x) \Gamma_{0}, \mathbf{B}(1) \Gamma_{1}, \ldots, \mathbf{B}(n) \Gamma_{n}$, where

$\forall x \mathbf{B}(x) \Gamma_{0}$ (denoted as $\boldsymbol{\Theta}_{\mathbf{1}}$ below) is empty or consists of formulas of the shape $\forall x_{j} \mathbf{B}\left(x_{j}\right) M_{j}, j \in\{1,2, \ldots\} ; \mathbf{B}(l) \Gamma_{l}, 1 \leq l \leq n$, is empty or consists of formulas of the shape $\mathbf{B}(l) C$; 
- $\exists \mathcal{B} \Delta$ denotes a list $\exists x \mathbf{B}(x) \Delta_{0}, \mathbf{B}(1) \Delta_{1}, \ldots, \mathbf{B}(n) \Delta_{n}$, where

$\exists x \mathbf{B}(x) \Delta_{0}$ is empty or consists of formulas of the shape $\exists x_{j} \mathbf{B}\left(x_{j}\right) N_{j}$, $j \in\{1,2, \ldots\} ; \mathbf{B}(r) \Delta_{r}, 1 \leq r \leq n$, is empty or consists of formulas of the shape $\mathbf{B}(r) D$;

- for every $i(i \in\{1,2\}) \mathbf{E B} \Pi_{i}\left(\mathbf{M B} \Delta_{i}\right)$ is empty or consists of formulas of the shape $\mathbf{E B}(A)(\mathbf{M B}(A)$, correspondingly).

A sequent $S$ is a reduced primary sequent, if $S$ is a primary one not containing MB $\Delta_{i}$ but $\Gamma, \Delta, \Pi_{1}, \Pi_{2}$ may contain modality $\mathbf{M B}$.

A reduced primary sequent $S$ is an EB-pure reduced primary one if $S$ is of the following shape $\Sigma_{1}, \Theta_{1}, \mathcal{B} \tilde{\Gamma}, \mathbf{E B} \Pi_{1} \rightarrow \Sigma_{2}, \mathbf{E B} \Pi_{2}$, where (1) $\Theta_{1}=$ $\forall x \mathbf{B}(x) \Gamma_{0}$; (2) $\mathcal{B} \tilde{\Gamma}$ is empty or denotes a list $\mathbf{B}(1) \Gamma_{1}, \ldots, \mathbf{B}(n) \Gamma_{n}$ such that $n$ is a number of agents and for every $l(1 \leq l \leq n) \mathbf{B}(l) \Gamma_{l}$ is not empty; (3) at least one from $\mathbf{E B} \Pi_{1}, \mathbf{E B} \Pi_{2}$ is not empty. Otherwise, the sequent $S$ is non- EB-pure reduced primary one.

From the shape of the primary sequent it is easy to see that bottom-up applying logical rules each sequent can be reduced to a set of primary sequents. As it follows from the shape of reduced primary sequent, bottom-up applying all reduction rules each primary sequent can be reduced to a set of reduced primary sequents.

To avoid loop-check in considered extension of the logic $K D 45_{n}$ let us introduce marks of two sorts and indices. The marks are used in separation rules for modalities $\mathbf{B}(t)$ and $\mathbf{E B}$. The first sort mark has the shape $\Upsilon^{*}\left(\Upsilon^{*} \in\right.$ $\left.\left\{\mathbf{B}^{*}(t), \mathbf{E B}^{*}, \mathbf{M B}^{*}\right\}\right)$. The first sort mark is defined as follows: let a formula $A$ is in the sphere of action of a marked modality $\boldsymbol{\Upsilon}^{*}$. Then an occurrence of any modality $\Upsilon(\boldsymbol{\Upsilon} \in\{\mathbf{B}(t), \mathbf{E B}, \mathbf{M B}\})$ in $A$ is marked by the first sort mark and $\mathbf{\Upsilon}^{* *}=\mathbf{\Upsilon}^{*}$. Both positive and negative occurrences of modality $\mathbf{\Upsilon}$ may contain the first sort mark. The second sort mark has the shape $\mathbf{B}^{-}(t)$. Only positive occurrences of belief modality $\mathbf{B}(t)$ in a sequent may contain the second sort mark. This mark is essential to get loop-check-free derivations in considered extension of the logic $K D 45_{n}$. Besides marked modalities we use indexed formulas of the shape $\exists x^{\circ} \mathbf{B}^{k}\left(x^{\circ}\right) A$, where $\exists x^{\circ} \in\{\varnothing, \exists x\}$ and $x^{\circ}=i$ if $\exists x^{\circ}=\varnothing$; an index $k$ is empty or $k \in\left\{*^{\circ} 1, \ldots, *^{\circ} m\right\}$, where $*^{\circ} \in$ $\{\varnothing, *\}$. Only positive occurrences of formulas of the shape $\exists x^{\circ} \mathbf{B}\left(x^{\circ}\right) A$ in the succedent of a sequent may contain the indices. In the index $k$ of the shape $*^{\circ} l l$ denotes a number of bottom-up applications of a separation rule for belief modality with the same main formula.

Let us introduce separation rule for everybody believes modality $\mathbf{E B}$. The conclusion of this separation rule is a EB-pure reduced primary sequent, such that logical part $\Sigma_{1} \rightarrow \Sigma_{2}$ is not derivable in the calculus $\log$. 
Separation rule $\left(S R_{1}\right)$ for everybody believes modality EB:

$$
\frac{\Theta_{1}^{*}, \Gamma_{0}, \mathcal{B}^{*} \tilde{\Gamma}, \tilde{\Gamma}, \mathbf{E B}^{*} \Pi_{1}, \Pi_{1} \rightarrow A^{\circ}}{\Sigma_{1}, \boldsymbol{\Theta}_{1}, \mathcal{B} \tilde{\Gamma}, \mathbf{E B} \Pi_{1} \rightarrow \Sigma_{2}, \mathbf{E B} \Pi_{2}, \mathbf{E B}\left(A^{\circ}\right)}\left(S R_{1}\right),
$$

where $\Theta_{1}$ and $\mathcal{B} \tilde{\Gamma}$ are determined in the definition of EB-pure reduced primary sequent; $\tilde{\Gamma}$ (obtained from $\mathcal{B} \tilde{\Gamma}$ ) denotes a list $\Gamma_{1}, \ldots, \Gamma_{n}$, where $n$ is a number of agents; $\mathbf{E B}\left(A^{\circ}\right) \in\{\varnothing, \mathbf{E B}(A)\}$; if $\mathbf{E B} \Pi_{2}, \mathbf{E B}\left(A^{\circ}\right)$ is empty, then $A^{\circ}$ is empty, otherwise $A^{\circ}=A$.

The formula $\mathbf{E B}(A)$ in the rule $\left(S R_{1}\right)$ is the main formula of this rule.

Let us introduce two separation rules for belief modality $\mathbf{B}(t)$ denoted as $\left(S R_{2}\right)$ and $\left(S R_{3}\right)$. The conclusion of these separation rules is a reduced primary sequent, such that logical part $\Sigma_{1} \rightarrow \Sigma_{2}$ is not derivable in $L o g$.

Separation rule $\left(S R_{2}\right)$ for belief modality $\mathbf{B}(t)$ :

$$
\frac{\Theta_{1}^{*}, \Gamma_{0}, \mathbf{B}^{*}(l) \Gamma_{l}, \Gamma_{l}, \mathbf{E B}^{*} \Pi_{1}, \Pi_{1} \rightarrow \boldsymbol{\Theta}_{\mathbf{2}}, \mathbf{B}(r) \Delta_{r}, \exists x^{\circ} \mathbf{B}^{\sigma}\left(x^{\circ}\right) M, M}{\Sigma_{1}, \forall \mathcal{B} \Gamma, \mathbf{E B} \Pi_{1} \rightarrow \Sigma_{2}, \exists \mathcal{B} \Delta, \exists x^{\circ} \mathbf{B}^{k}\left(x^{\circ}\right) M, \mathbf{E B} \Pi_{2}}\left(S R_{2}\right),
$$

where $\forall \mathcal{B} \Gamma, \exists \mathcal{B} \Delta$, and $\Theta_{1}$ are determined in the definition of primary sequent; $\Theta_{2}$ means $\exists x \mathbf{B}(x) \Delta_{0}$.

The formula $\exists x^{\circ} \mathbf{B}^{k}\left(x^{\circ}\right) M$ is the main formula of $\left(S R_{2}\right) ; \exists x^{\circ} \in\{\varnothing, \exists x\}$.

To define an index $\sigma$ let us consider two cases.

(1) $\exists x^{\circ}=\varnothing$, then $x^{\circ}=i$ and $\exists x^{\circ} \mathbf{B}^{k}\left(x^{\circ}\right) M$ has a shape $\mathbf{B}^{k}(i) M$. In this case $l=r=i$, i.e., $\mathbf{B}(l) \Gamma_{l}$ and $\mathbf{B}(r) \Delta_{r}$ consist of formulas of the shape $\mathbf{B}(i) D$. The index $\sigma$ is defined in the following way. Let $\rho(\eta)$ be the number of negative (positive, correspondingly) occurrences of modalities $\mathbf{B}(i), \mathbf{E B}, \mathbf{M B}$ in $M$; let $\tau_{0}, \tau_{1}, \ldots, \tau_{n}, \tau_{n+1}$ be the number of negative occurrences of modalities $\mathbf{B}(i), \mathbf{E B}, \mathbf{M B}$ in $\Gamma_{0}, \Gamma_{1}, \ldots, \Gamma_{n}, \Pi_{1}$, respectively, and $\tau=\max \left(\tau_{0}, \tau_{1}, \ldots\right.$, $\left.\tau_{n}, \tau_{n+1}\right), \rho^{\prime}=\max (\rho-\eta, \tau-\eta)$. Then $k \in\left\{*^{\circ} 0, \ldots, *^{\circ} \rho^{\prime}\right\}$ (where $*^{\circ} \in$ $\{\varnothing, *\})$, at the very beginning $k$ is empty and is treated as $*^{\circ} 0$. The index $\sigma$ is defined as follows: if $k=*^{\circ} l, l \in\left\{0, \ldots, \rho^{\prime}\right\}$ and $l<\rho^{\prime}$ then $\sigma=*^{\circ}(l+1)$; otherwise, i.e., if $k=*^{\circ} l$ and $l=\rho^{\prime}$, then $\sigma=-$.

(2) $\exists x^{\circ}=\exists x$. In this case all pairs consisting from $\mathbf{B}(l) \Gamma_{l}(1 \leq l \leq n)$ and $\mathbf{B}(r) \Delta_{r}(1 \leq r \leq n)$ must be reset. The index $\sigma$ is defined in the same way as in the case (1) replacing a modality $\mathbf{B}(i)$ with $\mathbf{B}(t)$, where $t$ is any agent variable or any agent constant.

The separation rule $\left(S R_{2}\right)$ corresponds to transitivity and Euclidean properties of belief modality.

Separation rule $\left(S R_{3}\right)$ for belief modality $\mathbf{B}(t)$ :

$$
\frac{\Theta_{1}^{*}, \Gamma_{0}, \mathbf{B}^{*}(l) \Gamma_{l}, \Gamma_{l}, \mathbf{E B}^{*} \Pi_{1}, \Pi_{1} \rightarrow}{\Sigma_{1}, \forall \mathcal{B} \Gamma, \mathbf{E B} \Pi_{1} \rightarrow \Sigma_{2}, \exists \mathcal{B} \Delta, \mathbf{E B} \Pi_{2}}\left(S R_{3}\right),
$$


where $\forall \mathcal{B} \Gamma, \exists \mathcal{B} \Delta$, and $\Theta_{1}$ are the same as in the rule $\left(S R_{2}\right)$.

The rule $\left(S R_{3}\right)$ corresponds to the serial property of belief modality.

During the reduction to primary and reduced primary sequents the following contraction rules are used.

Contraction rules. The rule allowing to replace $A, A_{1}$ with $A$ (where $A$ and $A_{1}$ coincide or are congruent ones [9]) is an ordinary contraction rule. The rules allowing to replace $\mathbf{B}^{k}(t) A, \mathbf{B}^{\circ}(t) A$, where $\circ \in\{\varnothing, *\}$, with $\mathbf{B}^{k}(t) A$, to replace $\mathbf{B}^{k}(t) A, \mathbf{B}^{-}(t) A$ with $\mathbf{B}^{-}(t) A$, and to replace $\Upsilon^{*} A, \boldsymbol{\Upsilon} A$, where $\mathbf{\Upsilon} \in\{\mathbf{B}(t), \mathbf{E B}, \mathbf{M B}\}$, with $\Upsilon^{*} A$ are marked contraction rules. Contraction rules are backward applied implicitly (together with other rules).

Some examples in next section demonstrate an application of the separation rules and the use of the marks/indexes.

\section{Description of Decision Algorithm}

In presented decision procedure for the extension of the logic $K D 45_{n}$ loopcheck-free sequent calculus is proposed. Such type calculi correspond to contraction-free calculus for modal logic. For a sequent containing different occurrences of mutual belief modality MB two kind of loop-check (saturation) are used: for positive occurrences of mutual belief modality loop-check is used to find non-logical (loop-type) axioms, and for negative ones loop-check (called degenerate saturation) is used to establish a non-derivability criterion.

So, along with the logical axioms, we use non-logical (loop-type) axioms (as in other works on temporal and agent-based logics with induction axioms, see, e.g., [12], [13]). First we define parametrically identical formulas and sequents. Namely, formulas $A$ and $A^{\prime}$ are called parametrically identical ones (in symbols $A \approx A^{\prime}$ ) if either $A=A^{\prime}$, or $A$ and $A^{\prime}$ are congruent [9], or differ only by the corresponding occurrences of eigen-constants of the rules $(\rightarrow \forall),(\exists \rightarrow)$; moreover, the occurrences of modality $\mathbf{\Upsilon}$ and marked modality $\mathbf{\Upsilon}^{*}$, where $\mathbf{\Upsilon} \in$ $\{\mathbf{B}(t), \mathbf{E B}, \mathbf{M B}\}$, are treated as coinciding. Sequents $S=A_{1}, \ldots, A_{k} \rightarrow$ $A_{k+1}, \ldots, A_{k+m}$ and $S^{\prime}=A_{1}^{\prime}, \ldots, A_{k}^{\prime} \rightarrow A_{k+1}^{\prime}, \ldots, A_{k+m}^{\prime}$ are parametrically identical (in symbols $S \approx S^{\prime}$ ), if $\forall j(1 \leq j \leq k+m)$ formulas $A_{j}$ and $A_{j}^{\prime}$ are parametrically identical ones. We say that a sequent $S=\Gamma \rightarrow \Delta$ subsumes a sequent $S^{\prime}=\Pi, \Gamma^{\prime} \rightarrow \Delta^{\prime}, \Theta$ (in symbols $S \succeq S^{\prime}$ ) if $\Gamma \rightarrow \Delta \approx \Gamma^{\prime} \rightarrow \Delta^{\prime}$ (in special case, $S=S^{\prime}$ ). A sequent $S^{\prime}$ is subsumed by $S$.

To obtain a negative criterion of derivability for the extension of the logic $K D 45_{n}$ let us introduce a notion of $b$-final sequent.

A primary sequent of the shape $\Sigma_{1}, \forall \mathcal{B}^{*} \Gamma, \mathbf{E B}^{*} \Pi_{1}, \mathbf{M B}^{*} \Delta_{1} \rightarrow \Sigma_{2}$, $\exists \mathcal{B}^{-} \Delta$ (in special case, $\Sigma_{2}, \exists \mathcal{B}^{-} \Delta$ is empty), such that logical part of this sequent, namely, $\Sigma_{1} \rightarrow \Sigma_{2}$ is not derivable in the calculus $L o g$, is $b$-final sequent. 
Let $D$ be a derivation in some calculus and $i$ be a branch in $D$. The primary sequent $S=\Gamma \rightarrow \Delta$ from the branch $i$ is a saturated sequent if, in the branch $i$ above $S$, there exists a subsumed by $S$ primary sequent $S^{\prime}$, i.e., $S \succeq S^{\prime}$.

Let $S=\Sigma_{1}, \forall \mathcal{B} \Gamma, \mathbf{E B} \Pi_{1}, \mathbf{M B} \Delta_{1} \rightarrow \Sigma_{2}, \mathbf{E B} \Pi_{2}$ be a saturated primary sequent in a derivation $D$. Then $S$ is degenerated saturated one if in $D$ there exists a subsumed by $S$ primary sequent $S^{\prime}$ of the shape $\Sigma_{1}^{\prime}, \forall \mathcal{B}^{*} \Gamma^{\prime}, \mathbf{E B}^{*} \Pi_{1}^{\prime}$, $\mathbf{M B}^{*} \Delta_{1}^{\prime} \rightarrow \Sigma_{2}^{\prime}, \mathbf{E B}^{\circ} \Pi_{2}^{\prime}(\circ \in\{\varnothing, *\})$ such that (1) logical part of $S^{\prime}$ is not derivable in the calculus $\log$; (2) $\Pi_{2}^{\prime}$ does not contain any positive occurrence of modality MB.

A saturated primary sequent $S$ is $\mathbf{M B}$ - saturated if $S=\Gamma \rightarrow \Delta, \mathbf{M B}(A)$. Sequents subsumed by an MB-saturated sequent will be used as non-logical axioms.

The decision algorithm for an arbitrary sequent is realized by means of a calculus for mutual belief $(M B Q)$.

Calculus $M B Q$ :

A calculus $M B Q$ is obtained from the calculus $\log$ adding the separation rules $\left(S R_{l}\right)(1 \leq l \leq 3)$, the reduction rules, contraction rules, and non-logical axioms of the shape $\Gamma \rightarrow \Delta, \mathbf{M B}(A)$.

A derivation $D$ in the calculus $M B Q$ is an ordered derivation, if it consists of several levels and each level consists of bottom-up applications of reduction rules. In this derivation at each level, when a set consisting of only reduced primary sequents is received, all possible bottom-up applications of the separation rules to every reduced primary sequent are realized. Each bottom-up application of the separation rules provides a possibility to construct a different (in general) ordered derivation $D_{k}(k \geq 1)$. Let in the level $j$ it be possible to bottom-up apply the rule $\left(S R_{2}\right)$ using as the main formula of this rule several formulas, namely, $\exists x_{1}^{\circ} B\left(x_{1}^{\circ}\right) M_{1}, \ldots, \exists x_{r}^{\circ} B\left(x_{r}^{\circ}\right) M_{r}$. In this case as the main formula of $\left(S R_{2}\right)$ we choose a such formula $\exists x_{i}^{\circ} B\left(x_{i}^{\circ}\right) M_{i}$ which was previously used as the main formula of this rule in the level $j-k(k \geq 1)$. A such tactic of construction of an ordered derivation is called directed one. To eliminate redundancy from constructed ordered derivation in each level we do not consider (for a while) a sequent which is subsumed by some sequent in the level.

The ordered derivation $D_{k}$ is a successful one, if each leaf of $D_{k}$ ends with axiom (either logical or non-logical). The notion of logical axiom is obvious. Let us consider the notion of non-logical axiom in more detail. Let in ordered derivation $D$ there exists reduction of a primary sequent of the shape $S=\Gamma \rightarrow$ $\Delta, \operatorname{MB}(A)$ to a set of primary sequents $S_{1}, \ldots, S_{p}$, where sequent $S_{k}(1 \leq$ $k \leq p)$ has the shape $\Pi, \Gamma_{k}^{\prime} \rightarrow \Theta, \Delta_{k}^{\prime}, \operatorname{MB}\left(A^{\prime}\right)$ and is such that $\Gamma \rightarrow \Delta \approx$ $\Gamma_{k}^{\prime} \rightarrow \Delta_{k}^{\prime}$ and $A \approx A^{\prime}$. The sequent $S$ belongs to $i$-th level of $D$ and $S_{k}$ belongs to $(i+l)$-th level of $D(l \geq 1)$. Then the sequents $S_{k}$ are considered as non- 
logical ( MB-loop-type) axioms of $M B Q$. In Section 5 it will be justified that non-logical axioms are founded automatically and consist of some parts of an end sequent of $D$.

If there exists an ordered derivation $D$ of sequent $S$ such that in a leaf of each branch $i$ of $D$ there is either a logical axiom, or a non-logical axiom, then in both these cases $M B Q \vdash S$ (positive criterion of termination of the procedure). If in all possible ordered derivations $D_{k}$ of a sequent $S$ there exists a branch having a sequent which is either non-derivable in $\log$ or degenerated saturated one or $b$-final one, then $M B Q \nvdash S$ (negative criterion of termination of the procedure).

In the next section it will be justify that for any sequent a process of construction of an ordered derivation always terminates and proceeds automatically.

Bottom-up application of the reduction rule $(\rightarrow \mathrm{MB})$ is induction-free one, if the left premise $S^{\prime}$ of this rule has a shape $\Gamma \rightarrow \Delta, \mathbf{E B}(A)$, where $\Delta, A$ do not contain positive occurrences of modality MB. If $M B Q \vdash^{D} S^{\prime}$ and $D$ does not contain non-logical axioms then this bottom-up application is successful.

From the notion of an ordered derivation in $M B Q$ we get the following

Lemma 1 (derivability criterion in $M B Q$ ) Let $S$ be an arbitrary sequent. Then $M B Q \vdash^{D} S$ if and only if each induction-free bottom-up application of the reduction rule $(\rightarrow \mathrm{MB})$ in $D$ is successful.

Let $\left(S R_{2}^{+}\right)$be the rule obtained from the rule $\left(S R_{2}\right)$ changing a definition of the index $\sigma$. Namely, let $\exists x^{\circ} \mathbf{B}^{k}\left(x^{\circ}\right) M$ be the main formula of the rule $\left(S R_{2}^{+}\right)$, and $k \in\left\{*^{\circ} 0, *^{\circ} 1, \ldots\right\}$ (where $*^{\circ} \in\{\varnothing, *\}$ ), at the very beginning $k$ is empty and is treated as $*^{\circ} 0$; if $k=*^{\circ} l$ then $\sigma=*^{\circ}(l+1)$. Let a calculus $M B Q^{+}$is obtained from $M B Q$ adding the rule $\left(S R_{2}^{+}\right)$. An application of the rule $\left(S R_{2}^{+}\right)$ in $M B Q^{+}$is degenerate if $\sigma \geq \rho^{\prime}+1$, where $\rho^{\prime}$ is determined in the same way as in the rule $\left(S R_{2}\right)$.

Analogously as in [13] using induction on number of the degenerate application of the rule $\left(S R_{2}^{+}\right)$we can prove

Lemma 2 If $M B Q^{+} \vdash S$ then $M B Q \vdash S$.

From Lemma 2 and relying on directed tactic in construction of ordered derivation we get

Lemma 3 Let $D$ be an ordered derivation in $M B Q$. Let $\Sigma_{1}, \forall \mathcal{B} \Gamma, \mathbf{E B} \Pi_{1} \rightarrow$ $\Sigma_{2}, \exists \mathcal{B} \Delta, \exists x^{\circ} \mathbf{B}^{k}\left(x^{\circ}\right) M, \mathbf{E B} \Pi_{2}$ be a conclusion of an application of the rule $\left(S R_{2}\right)$ in D. Then the same positive occurrence of the formula $\exists x^{\circ} \mathbf{B}^{k}\left(x^{\circ}\right) M$ may be the main formula of applications of the separation rule $\left(S R_{2}\right)$ in $D$ at most $\rho^{\prime}+1$ time, where $\rho^{\prime}$ is defined in the rule $\left(S R_{2}\right)$. 
Let us demonstrate saturation-free ordered derivations in $M B Q$, i.e., all branches of constructed ordered derivations end with logical axioms.

Example 1 (a) Let $S=\mathbf{B}(1) P \rightarrow \mathbf{B}(1) \mathbf{B}(1)(P \vee Q)$. We can bottom-up apply $\left(S R_{2}\right)$ or $\left(S R_{3}\right)$ to $S$. Bottom-up applying $\left(S R_{3}\right)$ to $S$ we get b-final sequent $\mathbf{B}^{*}(1) P, P \rightarrow$. Let us consider the possibility to bottom-up apply $\left(S R_{2}\right)$ to $S$. For $S$ we have $\rho=0, \eta=1, \tau=0$, and $\rho^{\prime}=0$. Therefore bottom-up applying $\left(S R_{2}\right)$ to $S$ we get $\sigma=-$ and $S_{1}=\mathbf{B}^{*}(1) P, P \rightarrow$ $\mathbf{B}^{-}(1) \mathbf{B}(1)(P \vee Q), \mathbf{B}(1)(P \vee Q)$. Again, we can bottom-up apply $\left(S R_{2}\right)$ or $\left(S R_{3}\right)$ to $S_{1}$. Let us apply $\left(S R_{2}\right)$. We can bottom-up apply $\left(S R_{2}\right)$ to $S_{1}$ only with $\mathbf{B}(1)(P \vee Q)$ as the main formula. Since for $S_{1} \rho^{\prime}=0$, we get $\sigma=-$ and $S_{2}=\mathbf{B}^{*}(1) P, P \rightarrow \mathbf{B}^{-}(1) \mathbf{B}(1)(P \vee Q), \mathbf{B}^{-}(1)(P \vee Q), P \vee Q$. Bottom-up applying $(\rightarrow \vee)$ to $S_{2}$ we get an axiom. Therefore $M B Q \vdash S$.

(b) Let $S=\mathbf{B}(1) \mathbf{E B}(P) \rightarrow \mathbf{B}(1)(P \vee Q)$, i.e., for $S \rho=0, \eta=0, \tau=1$, and $\rho^{\prime}=1$. Therefore bottom-up applying $\left(S R_{2}\right)$ to $S$ we get $\sigma=1$ and $S_{1}=$ $\mathbf{B}^{*}(1) \mathbf{E B}^{*}(P), \mathbf{E B}(P) \rightarrow \mathbf{B}^{1}(1)(P \vee Q),(P \vee Q)$. Since for $S_{1} \rho^{\prime}=1$ and $k=1$, bottom-up applying $\left(S R_{2}\right)$ to $S_{1}$ we get $S_{2}=\mathbf{B}^{*}(1) \mathbf{E B}^{*}(P), \mathbf{E B}^{*}(P)$, $P \rightarrow \mathbf{B}^{-}(1)(P \vee Q), P \vee Q$. Bottom-up applying $(\rightarrow \vee)$ to $S_{2}$ we get an axiom. Therefore $M B Q \vdash S$.

(c) Let $S=\mathbf{B}(1) \mathbf{E B}(\mathbf{B}(1) P) \rightarrow \mathbf{B}(1) A$, where $A=\neg \mathbf{B}(2) Q \vee P$. For $S$ we have $\rho=0, \eta=0, \tau=2$, and $\rho^{\prime}=2$. Therefore bottom-up applying $\left(S R_{2}\right)$ to $S$ we get $\sigma=1$ and, after applying $(\rightarrow \vee),(\rightarrow \neg)$, we get $S_{1}=\mathbf{B}^{*}(1) \mathbf{E B}^{*}\left(\mathbf{B}^{*}(1) P\right), \mathbf{E B}(\mathbf{B}(1) P), \mathbf{B}(2) Q \rightarrow \mathbf{B}^{1}(1) A^{*}, P$. Since for $S_{1} \quad \rho^{\prime}=2$, bottom-up applying $\left(S R_{2}\right)$ and $(\rightarrow \vee),(\rightarrow \neg)$ from $S_{1}$ we get $\sigma=2$ and $S_{2}=\mathbf{B}^{*}(1) \mathbf{E B}^{*}\left(\mathbf{B}^{*}(1) P\right), \mathbf{E B}^{*}\left(\mathbf{B}^{*}(1) P\right), \mathbf{B}(1) P, \mathbf{B}(2) Q \rightarrow$ $\mathbf{B}^{2}(1) A, P$. For $S_{2}$ we get again $\rho^{\prime}=2$. Bottom-up applying $\left(S R_{2}\right),(\rightarrow \vee)$, $(\rightarrow \neg)$ from $S_{2}$ we get $\sigma=-$ and $S_{3}=\mathbf{B}^{*}(1) \mathbf{E B}^{*}\left(\mathbf{B}^{*}(1) P\right), \mathbf{E B}^{*}\left(\mathbf{B}^{*}(1)\right.$ $P), \mathbf{B}^{*}(1) P, P, \mathbf{B}(2) Q \rightarrow \mathbf{B}^{-}(1) A, P$. Since $S_{3}$ is an axiom, $M B Q \vdash S$.

(d) Let $\{1, \ldots, n\}$ be a set of agent constants and $S=\mathbf{B}(1) P_{1}$, $\ldots \mathbf{B}(n) P_{n} \rightarrow \mathbf{E B}\left(\bigvee_{i=1}^{n} P_{i}\right)$. Bottom-up applying $\left(S R_{1}\right)$ and then $(\rightarrow \vee)$ we get an axiom. Therefore $M B Q \vdash S$.

(e) Let $\{1,2\}$ be a set of agent constants and $S=\mathbf{B}(1) P \rightarrow \mathbf{E B}(P \vee$ $\neg \mathbf{B}(2) P)$. Bottom-up applying $(\rightarrow \mathbf{E B}),(\rightarrow \wedge)$ from $S$ we get reduced primary sequents $S_{1}=\mathbf{B}(1) P \rightarrow \mathbf{B}(1)(P \vee \neg \mathbf{B}(2) P)$ and $S_{2}=\mathbf{B}(1) P \rightarrow$ $\mathbf{B}(2)(P \vee \neg \mathbf{B}(2) P)$. Bottom-up applying $\left(S R_{2}\right)$ and $(\rightarrow \vee)$ from $S_{1}$ we get an axiom. Bottom-up applying $\left(S R_{2}\right)$ and $(\rightarrow \vee),(\rightarrow \neg)$ from $S_{2}$ we get $S_{3}=\mathbf{B}(2) P \rightarrow \mathbf{B}^{1}(2)(P \vee \neg \mathbf{B}(2) P), P$. Bottom-up applying $\left(S R_{2}\right)$ and $(\rightarrow \vee)$ from $S_{3}$ we get an axiom. Therefore $M B Q \vdash S$. 
Let us demonstrate negative criterion of termination, i.e., construction of ordered derivations in $M B Q$ containing a branch which ends with $b$-final sequent or containing a degenerated saturated primary sequent.

Example 2 (a) Let $S=\rightarrow \exists x \mathbf{B}(x) A$, where $A=\neg \mathbf{E B}(P) \vee Q$, i.e., for $S$ $\rho=1$ and $\rho^{\prime}=1$. Bottom-up applying $\left(S R_{2}\right)$, and then $(\rightarrow \vee),(\rightarrow \neg)$ from $S$ we get $S_{1}=\mathbf{E B}(P) \rightarrow \exists x \mathbf{B}^{1}(x) A, Q$. Since for $S_{1} \quad k=\rho^{\prime}=1$, bottom-up applying $\left(S R_{2}\right)$, and then $(\rightarrow \vee),(\rightarrow \neg)$ from $S_{1}$ we get $S_{2}=\mathbf{E B}^{*}(P), P \rightarrow$ $\exists x \mathbf{B}^{-}(x) A, Q . S_{2}$ is not an axiom and is b-final. Therefore $M B Q \nvdash S$.

(b) Let $S=\mathbf{E B}(P), \mathbf{M B}(A) \rightarrow \mathbf{E B}(Q)$, where $A=P \supset \neg \mathbf{E B}(Q)$. Bottom-up applying $(\mathrm{MB} \rightarrow)$ to $S$ we get $S_{1}=\mathbf{E B}(P), \mathbf{E B}(\mathbf{M B}(A))$, $\mathbf{E B}(A) \rightarrow \mathbf{E B}(Q)$. Bottom-up applying $\left(S R_{1}\right)$ to $S_{1}$ we get $S_{2}=\mathbf{E B}^{*}(P), P$, $\mathbf{E B}^{*}(A), A, \mathbf{E B}^{*}\left(\mathbf{M B}^{*}(A)\right), \mathbf{M B}^{*}(A) \rightarrow Q$. Bottom-up applying $(\supset \rightarrow)$, $(\neg \rightarrow)$ from $S_{2}$ we get an axiom (with $P$ as the main formula) and $S_{3}=$ $\mathbf{E B}^{*}(P), P, \mathbf{E B}^{*}(A), \mathbf{E B}^{*}\left(\mathbf{M B}^{*}(A)\right), \mathbf{M B}^{*}(A) \rightarrow \mathbf{E B}^{*}(Q), Q$. Since $S \succeq$ $S_{3}$, from the shape of $S_{3}$ we get that $S$ is a degenerated saturated sequent. Therefore $M B Q \nvdash S$.

Let $S^{\prime}$ be a sequent obtained from the sequent $S$ replacing the formula $A$ by $P \supset \neg \mathbf{B}(1) Q$. Then we get derivation ending with a $b$-final sequent.

Let us demonstrate a derivation in $M B Q$ with $M B$-saturation, i.e., a constructed ordered derivation contains non-logical axioms along with logical ones.

Example 3 Let $S$ be $\mathbf{E B}(\forall x \mathbf{B}(x) P), \mathbf{M B}(A) \rightarrow \mathbf{M B}(\exists x \mathbf{B}(x) P)$, where $A=\exists x \mathbf{B}(x) P \supset \mathbf{E B}(\forall x \mathbf{B}(x) P)$. The sequent $S$ is a modified version of induction axiom for modality $\mathbf{M B}$.

Bottom-up applying $(\rightarrow \mathbf{M B})$ to $S$ we get two sequents $S_{1}=\mathbf{E B}(\forall x \mathbf{B}(x) P)$, $\mathbf{M B}(A) \rightarrow \mathbf{E B}(\exists x \mathbf{B}(x) P)$ and $S_{2}=\mathbf{E B}(\forall x \mathbf{B}(x) P), \mathbf{M B}(A) \rightarrow \mathbf{E B}$ $(\mathbf{M B}(\exists x \mathbf{B}(x) P))$. Bottom-up applying $(\mathbf{M B} \rightarrow)$ to $S_{1}$ we get the sequent $S_{1}^{\prime}=\mathbf{E B}(\forall x \mathbf{B}(x) P, \mathbf{E B}(A), \mathbf{E B}(\mathbf{M B}(A)) \rightarrow \mathbf{E B}(\exists x \mathbf{B}(x) P)$. Bottomup applying $\left(S R_{1}\right)$ to $S_{1}^{\prime}$ we get the sequent $S_{1}^{\prime \prime}=\forall x \mathbf{B}(x) P, A, \mathbf{M B}(A), \Delta \rightarrow$ $\exists x \mathbf{B}(x) P$, where $\Delta=\mathbf{E B}^{*}\left(\forall x \mathbf{B}^{*}(x) P\right), \mathbf{E B}^{*}(A), \mathbf{E B}^{*}\left(\mathbf{M B}^{*}(A)\right)$. Bottomup applying $(\supset \rightarrow)$ from $S_{1}^{\prime \prime}$ we get sequents $S_{11}=\forall x \mathbf{B}(x) P, \mathbf{M B}(A), \Delta \rightarrow$ $\exists x \mathbf{B}(x) P$ and $S_{12}=\forall x \mathbf{B}(x) P, \mathbf{E B}(\forall x \mathbf{B}(x) P), \mathbf{M B}(A), \Delta \rightarrow \exists x \mathbf{B}(x) P$. Since $S_{11} \succeq S_{12}$, at first we consider the sequent $S_{11}$. Bottom-up applying $(\mathbf{M B} \rightarrow)$ from $S_{11}$ we get $S_{11}^{\prime}=\forall x \mathbf{B}(x) P, \Delta \rightarrow \exists x \mathbf{B}(x) P$. Bottom-up applying $\left(S R_{2}\right)$ to $S_{11}^{\prime}$ we get an axiom with $P$ as the main formula. Therefore $M B Q \vdash S_{11}$. Since $S_{11} \succeq S_{12}, M B Q \vdash S_{12}$ as well.

Now let us consider the sequent $S_{2}$. Bottom-up applying $(\mathrm{MB} \rightarrow)$ to $S_{2}$ we get $S_{2}^{\prime}=\mathbf{E B}(\forall x \mathbf{B}(x) P), \mathbf{E B}(A), \mathbf{E B}(\mathbf{M B}(A)) \rightarrow \mathbf{E B}(\mathbf{M B}(\exists x \mathbf{B}(x) P))$. Bottom-up applying $\left(S R_{1}\right)$ to $S_{2}^{\prime}$ we get the sequent $S_{2}^{\prime \prime}=\forall x \mathbf{B}(x) P, A, \mathbf{M B}$ 
$(A), \Delta \rightarrow \mathbf{M B}(\exists x \mathbf{B}(x) P)$. Bottom-up applying $(\supset \rightarrow)$ to $S_{2}^{\prime \prime}$ we get two sequents $S_{21}=\forall x \mathbf{B}(x) P, \mathbf{M B}(A), \Delta \rightarrow \exists x \mathbf{B}(x) P, \mathbf{M B}(\exists x \mathbf{B}(x) P)$ and $S_{22}=\forall x \mathbf{B}(x) P, \mathbf{E B}(\forall x \mathbf{B}(x) P), \mathbf{M B}(A), \Delta \rightarrow \mathbf{M B}(\exists x \mathbf{B}(x) P)$. Since $S \succeq S_{22}, S$ is MB-saturated sequent. Now let us consider the sequent $S_{21}$. Bottom-up applying $(\rightarrow \mathrm{MB})$ and then $(\mathrm{MB} \rightarrow),\left(S R_{2}\right)$ in both branches of $(\rightarrow \mathrm{MB})$ we get an axiom with $P$ as the main formula. Therefore $M B Q \vdash S$.

\section{Foundation of Presented Decision Procedure}

To justify the presented decision procedure, we must found: (1) termination of the procedure, (2) invertibility of reduction and contraction rules, and existential invertibility (see below) of the separation rules in $M B Q$ and (3) MB-type saturated sequents as non-logical axioms. The termination will be founded by means of finiteness of the so-called $R$-subformulas of primary sequents which are generated during the construction of an ordered derivation. Let us define the notion of $R$-subformulas of a sequent.

Let $S$ be a primary sequent and $C$ be a formula entering $S$. A set of $R$ subformulas of $C$ from $S$ is denoted as $R S u b(C)$ and defined inductively.

1. $\operatorname{RSub}(P)=\varnothing$, where $P$ is a logical formula.

2. $R \operatorname{Sub}(\mathbf{E B}(A))=R \operatorname{Sub}(A)$.

3. $R S u b(\neg A)=R \operatorname{Rub}(A)$.

4. $R S u b(A \odot B)=\{R S u b(A)\} \cup\{R S u b(B)\}$, where $\odot \in\{\supset, \wedge, \vee\}$.

5. $R S u b(\mathbf{B}(i) A)=\{\mathbf{B}(i) A\} \cup\{R S u b(A)\}$.

6. $R S u b(\mathbf{M B}(A))=\{\mathbf{E B}(\mathbf{M B}(A))\} \cup\{R S u b(\mathbf{E B}(A))\}$.

7. $R \operatorname{Sub}(Q x \mathbf{B}(x) A)=R \operatorname{Sub}(\mathbf{B}(c) A)$, where $Q$ is $\forall(\exists)$ and $Q$ occurs positively (negatively) in $S, x$ is an agent variable and $c$ is a new agent constant.

8. $R \operatorname{Sub}(Q x \mathbf{B}(x) A)=R \operatorname{Rub}(A)$, where $Q$ is $\exists(\forall)$ and $Q$ occurs positively (negatively) in $S$.

A set of $R$-subformulas of a sequent $S=A_{1}, \ldots, A_{k} \rightarrow A_{k+1}, \ldots, A_{k+m}$ is denoted by $R S u b(S)$ and defined as $R S u b(S)=\cup_{i=1}^{k+m} R S u b\left(A_{i}\right) \cdot R^{*} \operatorname{Sub}(S)$ denotes a set obtained from $R S u b(S)$ by merging parametrically identical formulas.

From definition of $R^{*} S u b(S)$ we get that the set $R^{*} S u b(S)$ is finite. This fact (along with Lemma 3 ) is crucial to obtain termination of presented procedure.

Analyzing the construction of an ordered derivation in $M B Q$ we get that the presented decision procedure is exponential-time and PSPACE-complete, i.e., during the construction of an ordered derivation of a sequent $S$ we generate the primary sequents the length of which can be restricted by some polynomial depending on $R^{*} S u b(S)$. 
To justify the invertibility of reduction and contraction rules and the existential invertibility of the separation rules in $M B Q$ an infinitary calculus $M B Q_{\omega}$ is introduced. $M B Q_{\omega}$ is obtained from $M B Q$ by dropping the non-logical axiom, marks and indices in separation rules and replacing the reduction rule $(\rightarrow \mathrm{MB})$ by following infinitary rule:

$$
\frac{\Gamma \rightarrow \Delta, \mathbf{E B}(A) ; \ldots ; \Gamma \rightarrow \Delta, \mathbf{E B}^{k}(A) ; \ldots}{\Gamma \rightarrow \Delta, \mathbf{M B}(A)}\left(\rightarrow \mathbf{M B}_{\omega}\right),
$$

$k \in\{1, \ldots\} ; \mathbf{E B}^{1}(A)=\mathbf{E B}(A), \mathbf{E B}^{k}(A)=\mathbf{E B}\left(\mathbf{E B}^{k-1}(A)\right), k>1$.

Using induction on $\mathcal{O}(D)$, where $\mathcal{O}(D)$ is the height of a derivation $D$ [13] of the conclusion of a reduction rule in $M B Q_{\omega}$, we can prove an invertibility of reduction rules (including $\left.\left(\rightarrow \mathbf{M B}_{\omega}\right)\right)$ in $M B Q_{\omega}$.

Using reduction rules it is possible to construct a reduction of sequent $S$ to a set $\left\{S_{1}, \ldots, S_{m}\right\}$, where $S_{j}(1 \leq j \leq m)$ is a primary (reduced primary) sequent automatically. Using the invertibility of reduction rules we get that if $M B Q_{\omega} \vdash S$ then $M B Q_{\omega} \vdash S_{j}, j \in\{1, \ldots, m\}$.

It is easy to see that the separation rules $\left(S R_{l}\right)(l \in\{1,2,3\})$ are not invertible in the usual way but they are existential invertible. The separation rule $\left(S R_{l}\right)(l \in\{1,2,3\})$ is existential invertible if from derivability of the conclusion of the separation rule $\left(S R_{l}\right)$ follows that there exists at least one rule $\left(S R_{l}\right)(1 \leq l \leq 3)$ such that a premise of this rule is derivable. It is obvious that, in contrast to deterministic usual invertibility, existential invertibility is non-deterministic.

Using double induction on $\langle k(S), \mathcal{O}(D)>$, where $k(S)$ is a number of positive occurrences of modality $\mathbf{M B}$ in an end-sequent of the derivation $D$, we can prove an existential invertibility of separation rules.

Lemma 4 (existential invertibility of separation rules) Let $S$ be a reduced primary sequent, i.e., $S=\Sigma_{1}, \forall \mathcal{B} \Gamma, \mathbf{E B} \Pi_{1} \rightarrow \Sigma_{2}, \exists \mathcal{B} \Delta, \mathbf{E B} \Pi_{2}$, such that $M B Q_{\omega} \vdash S$ and $\log \nvdash \Sigma_{1} \rightarrow \Sigma_{2}$. Then either

- $S$ is an EB-pure reduced primary sequent and there exists a sequent $S_{1}$ such that $M B Q_{\omega} \vdash S_{1}=\mathbf{\Theta}_{1}^{*}, \Gamma_{0}, \mathcal{B}^{*} \tilde{\Gamma}, \tilde{\Gamma}, \mathbf{E B}^{*} \Pi_{1}, \Pi_{1} \rightarrow A^{\circ}$, where the sequent $S_{1}$ is defined in the formulation of the rule $\left(S R_{1}\right)$, or

- there exists a formula $\exists x^{\circ} \mathbf{B}^{k}\left(x^{\circ}\right) M$ from $\exists \mathcal{B} \Delta$, such that $M B Q_{\omega} \vdash S_{2}=$ $\mathbf{\Theta}_{1}^{*}, \Gamma_{0}, \mathbf{B}^{*}(l) \Gamma_{l}, \Gamma_{l}, \mathbf{E B}^{*} \Pi_{1}, \Pi_{1} \rightarrow \mathbf{\Theta}_{\mathbf{2}}, \mathbf{B}(r) \Delta_{r}, \exists x^{\circ} \mathbf{B}^{\sigma}\left(x^{\circ}\right) M, M$, where the sequent $S_{2}$ and the index $\sigma$ is defined in the formulation of the rule $\left(S R_{2}\right)$, or

- there exists $l \geq 0$ such that $M B Q_{\omega} \vdash S_{3}=\mathbf{\Theta}_{1}^{*}, \Gamma_{0}, \mathbf{B}(l)^{*} \Gamma_{l}, \Gamma_{l}, \mathbf{E B}^{*} \Pi_{1}$, $\Pi_{1} \rightarrow$, where the sequent $S_{3}$ is defined in the formulation of the rule $\left(S R_{3}\right)$. 
Using invertibility of the reduction and separation rules we can prove that the contraction rules are invertible in $M B Q_{\omega}$

Using Schütte method (analogously as in [8]) we get

Theorem 1 (soundness and $\omega$-completeness of $M B Q_{\omega}$ ) Let $S$ be a sequent. Then $\forall M=S \Longleftrightarrow M B Q_{\omega} \vdash S$. The cut rule is admissible in $M B Q_{\omega}$.

From the fact that $M B Q_{\omega} \vdash \mathbf{M B}(A) \equiv \mathbf{E B}(A) \wedge \mathbf{E B}(\mathbf{M B}(A))$ and admissibility of cut in $M B Q_{\omega}$ we get that the rule $(\rightarrow \mathbf{M B})$ is admissible and invertible in $M B Q_{\omega}$.

To get an equivalence between calculi $M B Q$ and $M B Q_{\omega}$ we introduce invariant calculus $I N M B Q$. To define this calculus let us introduce some auxiliary notions. Let $M B Q \vdash^{D} S$. Then a set of MB-saturated sequents, i.e., the sequents of the shape $\Gamma \rightarrow \Delta, \mathbf{M B}(A)$, in $D$ is denoted by $S a t\{S\}$. Let us decompose $S a t\{S\}$ into a set of sets $S a t^{i}\{S\}$ such that (1) $S a t\{S\}=\cup_{i} S a t^{i}\{S\}$; (2) $\forall i j\left(S a t^{i}\{S\} \cap \operatorname{Sat}^{j}\{S\}\right)=\varnothing$; (3) if $S_{1}, S_{2} \in \operatorname{Sat}^{i}\{S\}$, then $S_{1}, S_{2}$ have a common succedent member of the shape $\operatorname{MB}(A)$, which is called a nucleus of $S_{a t}{ }^{i}\{S\}$. Every set $S a t^{i}\{S\}$ is a component of decomposition of $S a t\{S\}$.

An invariant calculus $I N M B Q$ is obtained from the calculus $M B Q$ replacing the non-logical axioms by the following invariant rule:

$$
\frac{\Gamma \rightarrow \Delta, I ; I \rightarrow \mathbf{E B}(I) ; I \rightarrow \mathbf{E B}(A)}{\Gamma \rightarrow \Delta, \mathbf{M B}(A)}\left(\rightarrow \mathbf{M B}_{I}\right),
$$

where the invariant formula $I$ is constructed automatically.

The rule $\left(\rightarrow \mathbf{M B}_{I}\right)$ satisfies the following conditions:

- the conclusion of $\left(\rightarrow \mathbf{M B}_{I}\right)$, i.e., the sequent $S^{\prime}=\Gamma \rightarrow \Delta, \mathbf{M B}(A)$ is such that $S^{\prime} \in \operatorname{Sat}^{i}\{S\}$ and $\operatorname{Sat}^{i}\{S\}$ is $\left\{\Sigma_{i 1} \rightarrow \Pi_{i 1}, \mathbf{M B}(A) ; \ldots ; \Sigma_{i n} \rightarrow\right.$ $\left.\Pi_{\text {in }}, \mathbf{M B}(A)\right\}$, where $\operatorname{MB}(A)$ is the nucleus of $\operatorname{Sat}^{i}\{S\}$, i.e., $S^{\prime}$ is an MB-saturated sequent from a derivation of a sequent $S$ in $M B Q$;

- $I=\bigvee_{j=1}^{n}\left(\left(\exists \Sigma_{i j}\right)^{\wedge} \wedge \neg\left(\forall \Pi_{i j}\right)^{\vee}\right.$; let $\Pi$ be any set of formulas of the shape $\mathbf{B}\left(i_{1}\right) C_{1}, \ldots, \mathbf{B}\left(i_{m}\right) C_{m}$, where $\left.i_{l}(1 \leq l \leq m)\right)$ is an agent eigen-constant; then $Q \Pi=Q x_{1} \mathbf{B}\left(x_{1}\right) C_{1}, \ldots, Q x_{m} \mathbf{B}\left(x_{m}\right) C_{m}, Q \in\{\forall, \exists\}$ (therefore all the eigen-constants are correspondingly bounded); $\Gamma^{\wedge}\left(\Gamma^{\vee}\right)$ means the conjunction (disjunction, respectively) of formulas from $\Gamma$.

To prove that from $M B Q \vdash S$ follows $I N M B Q \vdash S$, a derivation of each MB-saturated sequent in $I N M B Q$ must be constructed.

Example 4 Let $S$ be the same sequent as in Example 3, i.e., has the shape $\mathbf{E B}(\forall x \mathbf{B}(x) P), \mathbf{M B}(A) \rightarrow \mathbf{M B}(\exists x \mathbf{B}(x) P)$, where $A=\exists x \mathbf{B}(x) P \supset$ 
$\mathbf{E B}(\forall x \mathbf{B}(x) P)$. From Example 3 it follows that $S$ is a MB-saturated sequent. From definition of the invariant formula $I$ we get $I=\mathbf{E B}(\forall x \mathbf{B}(x) P) \wedge$ $\mathbf{M B}(A)$. It is easy to verify that $L o g \vdash \mathbf{E B}(\forall x \mathbf{B}(x) P), \mathbf{M B}(A) \rightarrow I$ (1);

$$
I N M B Q \vdash I \rightarrow \mathbf{E B}(I) ; \quad(2) \quad I N M B Q \vdash I \rightarrow \mathbf{E B}(A) \quad \text { (3). }
$$

Applying $\left(\rightarrow \mathbf{M B}_{I}\right)$ to (1), (2) and (3) we get INMBQ $\vdash S$.

Analogously as in [13] we get

$$
M B Q \vdash S \Longleftrightarrow I N M B Q \vdash S \Longleftrightarrow M B Q_{\omega} \vdash S(*) \text {. }
$$

From $(*)$ we get that all reduction rules and contraction rules are invertible in $M B Q$ and the separation rules are existentially invertible in $M B Q$.

From Theorem 1 and (*) follows that $M B Q$ is sound and complete.

Using these facts, finiteness of $R^{*} S u b(S)$, and Lemma 3 we get the following

Theorem 2 Let $S$ be an arbitrary sequent. Then one can automatically construct a successful or unsuccessful ordered derivation $D$ of the sequent $S$ in $M B Q$ such that $D$ always terminates.

\section{References}

1. Alberucci, L., The modal $\mu$-calculus and the logic of common knowledge. Ph.D. thesis, Institut für Informatic and angewandte mathematik, Universität Bern, (2002).

2. Aldewereld, H., van der Hoek, W., Meyer, J.J.Ch., Rational teams: logical aspects of multiagent systems. Fundamenta Informaticae, 63(2-3), (2004), 159 - 183.

3. Dyckhoff, R., Contraction-free sequent calculi for intuitionistic logic, Journal of Symbolic Logic, 57, (1992), 795-807.

4. Fagin, R., Halpern, J.Y., Moses, Y., Vardi, M.Y., Reasoning about knowledge, MIT Press, Cambridge, Mass. (1995).

5. Fitting M., Thalmann L., Voronkov A., Term-modal logics. Studia Logica, 69(1), (2001), 133-169.

6. Halpern, J.Y., Shore R.A., Reasoning about common knowledge with infinitely many agents. Information and Computation, 191 (2004), 1-40.

7. Hudelmaier, J., A contraction-free sequent calculus for $S 4$, in Proof Theory for Modal Logic, H. Wansing, Ed. Kluwer Academic Publishers, Dordrechts, Boston/London (1996), 3-16.

8. Kawai, H., Sequential calculus for a first-order infinitary temporal logic. Zeitchr. für Math. Logic and Grundlagen der Math., 33, (1987), 423-432.

9. Kleene, S.C., Introduction to metamathematics, D.Van Nostrand Company, North-Holland Publishing Co., P. Noordhoff LTD (1952).

10. Lomuscio A., Colombetti M., QLB: A quantified logic for belief. Lecture Notes in Artificial Intelligence, 1193, (1996), 71-85.

11. Meyer, J.J.Ch., van der Hoek, W., Epistemic Logic for AI and Computer Science. Cambridge University Press, Cambridge, 1995.

12. Nide, N., Takata, S., Deduction systems for BDI logics using sequent calculus. In Proc. of AAMAS'02, (2002), 928-935.

13. Pliuškevičius, R., Pliuškevičienè, A., Decision procedure for a fragment of temporal logic of belief and actions with quantified agent and action variables. Annals of Mathematics, Computing \& Teleinformatics, 1(2) (2004), 51-72.

14. Wooldridge, M., Reasoning about Rational Agents. The MIT Press (2000). 